\title{
Strates
}

STRATES Matériaux pour la recherche en sciences sociales

$12 \mid 2006$

Nouvelles tensions impériales et recompositions en Europe centrale, orientale, et CEI

\section{Où en est le débat de philosophie politique en Russie?}

\section{Andrei TYRSENKO}

\section{OpenEdition}

\section{Journals}

Édition électronique

URL : http://journals.openedition.org/strates/1682

DOI : $10.4000 /$ strates. 1682

ISSN : $1777-5442$

Éditeur

Laboratoire Ladyss

Édition imprimée

Date de publication : 31 décembre 2006

ISSN : 0768-8067

Référence électronique

Andrei TYRSENKO, « Où en est le débat de philosophie politique en Russie ?», Strates [En ligne], 12 |

2006, mis en ligne le 19 juillet 2007, consulté le 08 septembre 2020. URL : http://

journals.openedition.org/strates/1682; DOI : https://doi.org/10.4000/strates.1682

Ce document a été généré automatiquement le 8 septembre 2020.

Tous droits réservés 


\title{
Où en est le débat de philosophie politique en Russie?
}

\author{
Andrei TYRSENKO
}

1 En Russie, la philosophie politique est une discipline relativement récente. Elle n'existe guère que depuis 1992, soit depuis la disparition de l'Union soviétique. Grosso modo, la situation actuelle de cette discipline peut être caractérisée comme suit :

2 - malgré une certaine quantité de publications spécifiques, en Russie ce domaine des sciences humaines reste peu développé ;

3 - on constate une forte influence sur la formation en philosophie politique de la tradition occidentale ;

4 - le débat actuel touchant les problèmes de la philosophie politique se déroule en fonction de la vie politique russe et se réfère aux paradigmes traditionnellement élaborés par l'école occidentale.

5 En Russie, on entend par philosophie politique la discipline qui étudie les principes de l'autocréation politique de la société qui comprend l'ontologie et l'épistémologie de la chose politique ${ }^{1}$.

6 Dans le cadre de cet article, nous nous proposons de nous arrêter sur trois grands problèmes aujourd'hui vivement discutés en Russie: les rapports entre pouvoir et nature de l'État; les idéologies politiques; la modernisation dans le contexte de l'expérience nationale.

Rapports entre pouvoir et nature de l'État

7 Le pouvoir et l'État appartiennent traditionnellement au domaine d'étude des politologues. On parle souvent d'un « système russe ", qui serait caractéristique de la culture politique de la Russie. Le pouvoir autosuffisant de l'État est l'élément central de ce système ${ }^{2}$. Certes, l'État autoritaire a caractérisé toutes les étapes de l'histoire nationale. La notion même d' «État» désigne en russe une institution détenant la propriété des biens et des êtres humains. Le pouvoir politique en Russie n'a jamais été exercé en fonction de la société civile, celle-ci n'existant pas. Les philosophes de la politique et les juristes russes de la seconde moitié du XIX ${ }^{e}$ siècle, voire ceux de l'époque 
dite des grandes réformes libérales, avancèrent l'idée que la liberté politique est intimement liée à la rigueur de la loi et à la solidité de l'État ${ }^{3}$.

Aujourd'hui, la philosophie politique considère l'État et le pouvoir comme les éléments constitutifs du politique, tant dans le cadre du pays que dans le cadre international ${ }^{4}$.

Pour la philosophie politique russe, le pouvoir ne se confond pas avec l'influence et exprime une disposition ${ }^{5}$. Le fait du pouvoir consiste en l'assujettissement de la volonté de l'objet du pouvoir à la volonté du sujet ${ }^{6}$. Le pouvoir est ainsi interprété sous l'angle de la volonté de puissance. On distingue alors les rapports de pouvoir suivants: le pouvoir peut revêtir des formes de coercition, de contrainte, d'incitation, de conviction, de manipulation et d'autoritéf .

Dans la mesure où l'espace post-soviétique devient de plus en plus démocratique, la notion de " pouvoir » dans le discours politique russe perd progressivement sa position hiérarchique dominante pour devenir un facteur stabilisant dans des conditions de crise plutôt qu'une forme étatique unifiée ${ }^{8}$.

11 Actuellement, la philosophie politique russe procède à l'inventaire des voies possibles du renforcement du pouvoir de l'État et de son efficacité, dans le contexte de son affaiblissement consécutif à la période Eltsine.

Nous passerons en revue ici deux des solutions avancées au cours des discussions qui se déroulent actuellement sur le sujet.

La première variante est liée à l'évolution de l'État. Partis de la théorie de l'interractionnisme symbolique, ses tenants rappellent en tout premier lieu que le pouvoir se cristallise par l'effet de communications symboliques qui permettent, à leur tour, d'imposer la volonté des gouvernants aux gouvernés'. La solidarité civile, les institutions de la société civile qui avaient pu occuper l'espace entre gouvernants et gouvernés, tombent de plus en plus en désuétude. La société civile cède ses positions aux marchés politiques qui sont assimilés aux marchés économiques en vertu du principe du choix rationnel, sous-jacent à ces deux types de marchés. Les symboles du marché politique se présentent comme les images politiques venant remplacer les traditions, les idéologies et les rites dans leur rôle de repères de l'espace public.

Le processus de la création en Russie d'un marché politique se déroule dans des conditions fondamentalement différentes de celles qui prévalent en Occident. L'ayant constaté, nos spécialistes de la philosophie politique en Russie s'efforcent d'en administrer la preuve. Il suffit déjà de dire qu'il n'y a jamais eu en Russie d'institutions de la société civile et, en particulier, de mass média indépendants comme c'était le cas en Occident. À la différence de la Russie, les démocraties occidentales ont intégré des valeurs fondamentales comme l'«éthique» et l'«infrastructure morale " (essentiellement la loyauté, la confiance, la solidarité, la dignité des personnes et de leur travail $)^{10}$. Mais l'appel des uns à régénérer ces valeurs en Russie se heurte au scepticisme des autres. De l'avis de ces derniers, un autre avenir est à envisager pour la Russie, parce que ce pays n'est plus en état de suivre l'expérience de l'Occident après sa propre expérience des régimes totalitaires d'abord, puis autoritaires ${ }^{11}$ ensuite. Cela veut dire que l'élargissement inédit de la participation politique dans les sociétés dépourvues d'une culture politique développée, d'une part, et la multiplication des moyens de contrôle politique du comportement des acteurs, d'autre part, risquent de se solder par l'apparition d'un conformisme politique de masse. 
dant, le vecteur du processus politique actuel se définit dans la philosophie politique russe dans les termes suivants: l'éclipse progressive de mécanismes de la représentation sociale et politique (on peut évoquer les changements de statut des partis politiques, la perte d'intérêt pour les programmes et les idéologies, la substitution de l'image des hommes politiques à l'identification sociale, etc.) transforment le système de gouvernement représentatif en système de gouvernement par représentation ou autoreprésentation des gouvernants aux gouvernés. Avec cela, il est communément admis que le mécanisme sous-jacent de ce système est le marché politique. Les spécialistes ne font pas l'unanimité quant à une telle évolution politique. En particulier, certains tournent leur attention vers la théorie de la "société de réseau » (network society), à la recherche d'une alternative valable au modèle du marché politique et à celui de la hiérarchie à l'époque de la globalisation et de l'instabilité du développement social. La théorie des réseaux politiques prend ses racines dans les études consacrées à l'interaction de la société civile et de l'État, du gouvernement et des groupes d'intérêts. Elle construit un modèle spécifique de gestion politique et de prise de décisions ${ }^{12}$.

16 Dans cette optique, il convient de mettre au clair la thèse de la démocratie dirigée, très présente dans le débat politique en Russie. Celle-ci implique à la fois des procédés démocratiques et des procédés autoritaires. Une telle forme de pouvoir parvient à ses fins grâce à la mise en œuvre cumulée des ressources administratives, économiques, financières, informationnelles. Dès lors le régime de démocratie dirigée s'identifie à un régime de pouvoir personnel ${ }^{13}$.

17 Pour autant, l'économie libérale, l'orientation à l'Occident, les libertés individuelles se maintiendront, affirment les politologues russes. Le modèle de démocratie dirigée peut rendre l'État efficace au cas où, conformément aux adeptes de cette solution, on réussit à exercer le pouvoir en ayant recours aux nouvelles technologies politiques.

18 La seconde variante tient à la mise en pratique de l'idée d'empire. En Russie l'idée d'empire a émergé avec force dans la seconde moitié des années 1980. Dans le sillage de la philosophie politique occidentale, ce thème a fait un retour dans le débat politique. Les spécialistes des sciences politiques tentent de repenser l'héritage historique russe relatif au paradigme de l'empire. Plus spécialement, l'intérêt pour ce paradigme est suscité par les nouveaux défis auxquels les civilisations contemporaines sont confrontées. Si, auparavant, les empires engendraient des civilisations, maintenant ce sont les civilisations qui contribuent à faire apparaître des empires, ceci dans un contexte aggravé de conflits internationaux ${ }^{14}$. Plusieurs tendances en témoignent à l'échelle mondiale, comme le rejet répété du modèle de "l'État-nation » dans le procès d'intégration en cours, le recours aux valeurs occidentales, etc. La philosophie politique russe avance les éléments constitutifs d'une vision d'ensemble du paradigme de l'empire, qu'on peut résumer comme suit :

19 - présence dans le système de légitimation politique de composants universels tels que le pouvoir substantiel ou transcendantal, les élites universelles qui coexistent avec les élites traditionnelles, l'intégration des éléments culturels et ethniques ${ }^{15}$;

20 - tendance constante à l'extension territoriale ;

21 - pratique inexistante d'assimilation des ethnies intégrées au territoire de l'État, de leurs particularités culturelles et ethniques. Il s'agit d'une mosaïque multiculturelle sur le territoire de l'empire. 

civilisation. En même temps, le type d'identification politique orienté vers l'Étatnation, perdrait définitivement sa signification ${ }^{18}$.

31 Ainsi examinées les deux variantes d'organisation du pouvoir actuellement en discussion, je passerai au problème suivant. La philosophie politique russe d'aujourd'hui met l'accent sur l'impératif d'intégrité territoriale de l'État, autrement dit sur la dimension territoriale du pouvoir. Durant les deux crises politiques de 1991 et de 1993, les élites russes ont accepté le modèle fédératif de l'État. Or ce modèle n'avait jamais fonctionné auparavant. Avant la Révolution d'octobre, l'Empire russe évoluait vers l'État unitaire, régime où un seul pouvoir politique s'exerce sur l'ensemble du territoire ${ }^{19}$. La Fédération de Russie, partie intégrante de l'URSS, se présentait comme une république unitaire, dotée de larges autonomies régionales et municipales. Dans les commentaires à la Constitution de 1993, il est dit que la Russie s'est développée comme un État centralisé et unitaire. Le principe de la Fédération territoriale et nationale, adopté après 1917, était devenu l'instrument qui permettait de rompre l'isolement national. Ce principe correspondait à l'objectif politique du gouvernement soviétique, qui se proposait de mêler les nations et les ethnies pour constituer le peuple soviétique. Cette ligne politique était un des éléments de l'autoritarisme soviétique ${ }^{20}$. Dans la Russie post-soviétique, le principe territorial caractérise la politique des ethnoélites, préoccupées de renforcer leurs positions et leur pouvoir.

Jusque-là on peut constater l'accord unanime des spécialistes. Mais dès qu'il s'agit de trouver une solution pour la Russie, les divergences sont immédiates. Deux points de vue se dégagent généralement. 

culturelle et nationale laissant la place à des autonomies locales élargies. En lieu et place de la chambre haute de la Douma (le Conseil de la Fédération), on propose d'instituer un Conseil d'État à la manière de celui qui existait du temps de l'Empire russe - un organe corporatif composé de représentants de la haute administration et des autonomies locales ${ }^{21}$. G. Popov, un des leaders de la Perestroika, se prononce aussi pour le principe de l'autonomie culturelle et nationale pour la Russie. Il pense qu'un modèle qui ne comporterait pas de composante ethnique, tout comme le modèle territorial et national existant, ne répondent aux besoins de la Russie. Il souhaiterait créer auprès de chaque organe représentatif des Chambres des ethnies dotées de leurs propres budgets ${ }^{22}$.

34 L'autre point de vue table sur la sauvegarde de la forme fédérative de l'État. Dans son optique, le modèle de l'État-nation n'est pas applicable à la Russie. L'originalité nationale des nations peut être préservée, uniquement dans le cadre de la Fédération. Les partisans de cette option appellent à l'union des nations ${ }^{23}$. Ils associent la désintégration de l'Union soviétique à un processus à double sens. D'une part, le développement des nations, qu'ils appellent nations-ethnies, a amené celles-ci à se consolider au sein des républiques de l'Union soviétique. D'autre part, l'impératif politique d'unification et d'internationalisation tenait en échec la première tendance ${ }^{24}$. La Fédération de Russie rénovée est censée devenir une communauté de nationsethnies en même temps qu'un État-nation unique pour toutes les nations-ethnies. L'Assemblée des peuples de la Russie a défini la Fédération de Russie, non seulement comme une fédération de territoires, mais aussi comme une fédération de nations et d'ethnies unifiées autour du peuple russe. comme la clé de voûte de l'orientation sociale et de la structure de l'État russe ${ }^{25}$. Mais il convient de préciser ici la nature de cette notion traditionnelle dans la mentalité russe : la nation se conçoit comme le stade supérieur du développement de l'ethnie ${ }^{26}$.

De leur côté, les tenants de l'idée de l'empire estiment qu'une seule Fédération peut être fiable - la Fédération basée sur l'empire. Notons que ces deux formes d'État sont fort proches, et ceci pour deux raisons : d'abord, elles sont ethniquement hétérogènes ; ensuite, le pouvoir politique étant médiat, n'est pas direct.

Avec les débuts de la Présidence de Poutine, les politologues ont commencé à porter la discussion sur le concept de "fédéralisme exécutif». La création par Poutine de sept régions ayant à leur tête des représentants délégués en a été le prétexte. Quels sont ses traits caractéristiques? Tout d'abord, ce type de fédéralisme suppose un déplacement de l'accent politique de la procédure de conviction vers une procédure de soumission, voire vers un processus politique fermé. Ce type de fédéralisme, d'après certains politologues, permettrait de se rapprocher au plus près d'un modèle de fédéralisme idéa ${ }^{27}$. D'autant que le fédéralisme est réputé être la forme la mieux adaptée au règlement d'éventuels conflits ethniques ${ }^{28}$.

L'idéologie politique

38

Pendant longtemps l'idéologie communiste servit de facteur unificateur pour la société polyethnique soviétique. Depuis la chute de l'Union soviétique, la Russie reste plongée dans un vacuum idéologique évident. La nécessité s'impose donc de repenser le rôle des idéologies dans la vie de la société russe. Ainsi la dimension idéologique de la philosophie politique s'est-elle tout naturellement trouvée au cœur du débat actuel. La 
question est de savoir si les idéologies sont possibles dans le monde d'aujourd'hui. Trois positions émergent ici.

1. Conformément à la première, la politique, qui est conflictuelle de par sa nature, ne se conçoit pas sans idéologies. Il s'agit davantage de l'affirmation du pluralisme de courants idéologiques que de la dé-idéologisation du monde politique. Qu'on me permette de rappeler les éléments qui définissent l'idéologie, d'après les adeptes de ce point de vue. Les voici: a) le lien de l'idéologie avec la conception du monde de l'époque; $b$ ) les grandes orientations à caractère de programme, avancées à partir de cette conception du monde; c) la stratégie de la réalisation des dites orientations; d) leur propagande; e) les mesures concrètes pour la réalisation du programme idéologique $^{29}$. Au nombre des principaux courants idéologiques, on distingue le libéralisme, le conservatisme, le marxisme, le socialisme démocratique, les totalitarismes de gauche et de droite. Actuellement, les auteurs qui se consacrent à la philosophie politique discutent de l'évolution des mentalités et des idéologies en Russie. La question idéologique impliquerait deux solutions pour la Russie : soit, dans les têtes pensantes, la recherche d'une idée susceptible d'unir la population malgré sa grande diversité ; soit l'émergence de cette idée au sein de la société civile, au fur et à mesure de son mûrissement politique ${ }^{30}$. Malgré l'influence accrue en politique de facteurs non-idéologiques, j'en ai parlé plus haut, il existe beaucoup d'autres facteurs favorables au développement des idéologies. La situation politique en Russie exige le dialogue, un dialogue fondé sur le pluralisme idéologique. Le vacuum idéologique persistant, à un moment où il n'y a pas de consensus, au niveau des élites russes, sur les valeurs et les fins politiques, rendra la vie politique en Russie encore plus opaque et imperméable aux citoyens ${ }^{31}$. Certains spécialistes penchent en faveur d'une introduction de l'idéologie par le haut, afin de ne pas avoir à attendre que l'idéologie se développe d'en bas, ce qui risque de prendre du temps ${ }^{32}$. D'après eux, la Russie a besoin d'une idéologie formulée et d'une structure idéologique définie pour encadrer le processus des réformes et les rendre plus efficaces ${ }^{33}$.

2. La seconde position s'appuie, dans une large mesure, sur l'approche accentuant l'aspect communicationnel du politique à l'époque post-moderne. Il en ressort que dans la société post-moderniste les idéologies cèdent la place aux technologies politiques créées sur le marché politique ${ }^{34}$. Le pragmatisme politique, l'adaptation au contexte, aux acteurs précis, deviennent le principal critère de la politique.

41 En Russie, compte tenu de la diversité de formes du discours politique, en raison des nombreux points de vue sur les transformations sociales et politiques en cours, il serait pratiquement impossible de faire l'impasse sur les idéologies politiques. C'est pourquoi la plupart des théoriciens russes insistent sur la priorité à donner aux idéologies, pour assurer l'intégration efficace de la société. Sont envisagées deux solutions qu'il paraît logique d'aborder sous l'angle de la communication politique : convient-il de fonder la communication politique sur le projet idéologique et de rendre ainsi possible l'intégration politique de la société russe ? Ou faut-il constituer le discours politique à partir de la propagande politique, voire des images politiques, pour promouvoir les projets qui possèdent leur propre potentiel d'unification politique de la société ${ }^{35}$ ? La réponse à ce dilemme dépend dans une large mesure du type de pouvoir qui va s'instaurer en Russie. Autrement dit, tout ce qui est tenté aujourd'hui pour activer la participation politique de la population sur une base idéologique, se trouve inévitablement confronté à la question de la production des images politiques, au 
recours aux technologies informatiques pour assurer le soutien d'un projet. Une telle orientation suppose l'amélioration des technologies informatiques, le renforcement de l'État en tant qu'un des principaux acteurs du marché informatique, ceci aux dépens de l'État-nation. Bien sûr, la conscience politique russe est à caractère nettement idéologique, ce qui permet de trouver plus aisément le soutien idéologique adéquat de l'organisation de la communication entre les gouvernants et les gouvernés. Mais d'autres facteurs, telles l'intégration de la Russie aux structures globales de la société de réseau (network society), la mise en place de la verticale informatique, conduisent à la consolidation du pouvoir médiatique et au déclin des idéologies. Si néanmoins on peut parler de la présence des idéologies dans la vie politique russe, il s'agira des idéologies qui n'ambitionnent pas de fonctions globales ${ }^{36}$.

3. Enfin suit la troisième attitude à l'égard de l'idéologie. Faute d'idéologie dominante définissant la conception du monde, les adeptes de cette attitude tiennent bien compte des approches de la sociolinguistique modernisée. Ils partent de la représentation d'une nature sémiotique des événements sociaux. D'après eux, la description du social est celle d'une forme de signe dont sont revêtues les interractions sociales. Des changements spectaculaires ont eu lieu dans l'approche sociolinguistique traditionnelle qui postulait la primauté de l'étude des interactions des langues liées aux contacts inter-ethniques et à la différenciation sociale de la langue. Il est admis d'une part que la société influence non seulement les structures formelles de la langue, mais aussi celles du fond, donc sa sémantique. D’autre part, le mécanisme idéologique étant tombé en désuétude, c'est la langue qui conditionne considérablement les structures sociales car elles constituent un système de connaissance forgeant dans une large mesure une représentation ethnique de la société à partir d'une conception linguistique du monde ${ }^{37}$.

Le débat actuel autour du rôle des idéologies dans la vie politique russe est nourri dans une large mesure par leur état présent. Il faut parler en effet d'idéologies n'existant encore que sous une forme rudimentaire.

Au niveau officiel, le thème de "l'idée nationale " est apparu lors des dernières élections présidentielles. La recherche de "l'idée russe » est communément liée à une crise d'identité nationale. Pour la première fois, l'idée a été formulée lors des crises d'identité survenues à la charnière des $\mathrm{XIX}^{\mathrm{e}}$ et $\mathrm{xx}^{\mathrm{e}}$ siècles. Le contenu de l'idée nationale se définit différemment. Dans le programme Poutine, les éléments de "l'idée russe » se présentaient comme le renforcement de l'État-nation, le renouveau patriotique, la solidarité sociale dans le cadre des valeurs humaines universelles. Mais on peut suggérer deux autres définitions de "l'idée russe ", l'une démocratique, la seconde conservatrice. La démocratique envisage les éléments suivants : la tradition historique, l'aménagement du territoire, la culture "éclairée», la démocratie. La version conservatrice de "l'idée russe $»^{38}$ aurait ses sources dans l'héritage byzantin, qui ressurgit sous les trois formes géopolitiques (l'Est contre l'Ouest), sociale (la communauté des travailleurs) et politique (le renforcement de l'État ou le retour à l'empire $\left.{ }^{39}\right)$. Avec cela, le courant néo-eurasiatique, en développant la tradition eurasiatique classique, propose d'organiser la communauté eurasiatique sur la base d'une synthèse super-ethnique et super-confessionnelle ${ }^{40}$. Le renouveau du courant eurasiatique à partir du 1985 actualisa la théorie politique de L. Goumilev. Pour les adeptes du néo-eurasisme, l'impact de l'héritage intellectuel de Goumilev sur les débats politiques en cours se traduit par trois moments. On met en relief la théorie de la 
passion comme le développement de l'« idéalisme eurasiatique». Ensuite vient la focalisation de l'attention scientifique sur la préhistoire des "empires nomades" orientaux et la découverte de l'héritage ethnique et culturel majeur des autochtones de l'ancienne Eurasie. Enfin, on souligne le mérite qu'a Goumilev d'approfondir l'approche turcophile de la théorie « de la complémentarité ethnique ${ }^{41}$ ».

D'autre part, force est de constater l'intérêt que suscite l'idéologie du socialisme démocratique ${ }^{42}$. Le libéralisme, de même, garde aussi un certain nombre d'adeptes, qui proposent de refaire le consensus social sur la base des valeurs libérales.

À l'heure actuelle, on discute vivement la question de savoir s'il est envisageable, sur la base de "l'idée russe " rénovée, d'effectuer une synthèse du libéralisme et du socialisme démocratique ${ }^{43}$.

La modernisation de la Russie

Le problème de la modernisation, qui fut capital pour la Russie des années 1990, est toujours d'actualité aujourd'hui. Dans le même temps, là encore, il faut constater l'absence de consensus parmi les spécialistes russes. Le modèle binaire, qui envisage la dichotomie «modernisation organique versus modernisation non organique » (c'est le cas de la Russie) n'est plus au cœur des débats. Les spécialistes sont passés à un autre modèle, supposant trois types de modernisation :

- la modernisation endogène qui se fait sur sa propre base (Europe, États-Unis) ;

49 - la modernisation de type endogène-exogène qui s'effectue aussi bien sur sa propre base qu'avec des éléments empruntés (Russie, Turquie, Grèce) ;

- la modernisation exogène qui est orientée vers les emprunts extérieurs ${ }^{44}$.

51 Pour les auteurs qui prônent la théorie rationaliste de la modernisation, ce processus a pour but d'opérer en Russie la transition de la société traditionnelle vers la société moderne, ainsi que l'instauration de la démocratie. Conscients cependant du fait que la modernisation n'est pas un processus linéaire, ils envisagent la mise en place de nouvelles institutions et la transformation des institutions traditionnelles ${ }^{45}$.

52 Un modèle fort intéressant, fondé sur l'expérience des pays occidentaux, a été avancé par M. Ilyine. Ce modèle comprend trois phases :

53 - la première Modernité, phase initiale d'une formation du type de l'État-nation, avec société civile et naissance du droit constitutionnel ;

54 - la Modernité moyenne, marquée par la différentiation de la société civile et de l'État et par l'apparition d'un système de représentation politique ;

55 - la Modernité accomplie, où se met en place un mécanisme approprié de changement du cours politique et des discours politiques, en conformité avec les votes aux élections. Mais comme l'affirme l'auteur de ce modèle, la Modernité accomplie doit aménager la consolidation ultérieure de la démocratie au regard de quelques segments encore discordants. Ceux-ci tiennent essentiellement aux niveaux géopolitique, culturel, de civilisation, de maturité de la nation. C'est à la nation qu'il incombe d'opérer l'intégration à l'État-nation et à la société civile de la substance culturelle et des fonctions civilisatrices. Toutefois, cette phase est encore à venir ${ }^{46}$.

56 M. Ilyine a étendu son modèle à la Russie. Ici, le type de modernisation impose l'application parallèle d'institutions traditionnellement russes et d'autres, empruntées à l'extérieur. L'auteur regroupe les institutions russes en quatre blocs :

57 - le bloc seigneurial ou patrimonial (c'est-à-dire l'autorité familiale); 

perpétuel. Il convient encore de retenir qu'en vertu de la faiblesse de la société civile, la modernisation en Russie ne visait que l'État. contradictoire et alternatif. En même temps, ces chercheurs sont unis par un pari sur l'universalisme et le rationalisme du développement politique, même si les principes de l'universalisme se combinent chez eux avec des éléments de particularisme et que de la synthèse des uns et des autres dépend le succès de la modernisation.

dit, il y a des spécialistes pour mettre en doute le concept même de modernisation. Ceux-là critiquent les approches essentialistes de la théorie du changement des régimes politiques. L'approche structuraliste, qui se réfère à l'influence des structures (d'ordre économique, culturel, social) sur la modernisation politique, est jugée insuffisante : des structures comparables produisent des effets politiques différents. D'autre part, du fait qu'elle se concentre sur l'action des acteurs, l'approche procédurière serait propre, semble-t-il, à rectifier les insuffisances de l'approche structuraliste. Mais si cette approche peut expliquer comment s'effectue la transition à la démocratie, elle n'est pas à même de répondre à la question de savoir pourquoi la transition a lieu.

64 Le courant critique qui se manifeste aujourd'hui en philosophie politique met l'accent sur l'impossibilité où l'on est d'affirmer que la modernisation des régimes postsoviétiques conduira obligatoirement à la démocratie. D'après un des représentants de ce courant, la seule chance de la démocratie tient au développement de la concurrence politique et des institutions officielles. La différence entre les institutions officielles et informelles est vue de la manière suivante : les institutions officielles sont les organes des pouvoirs législatif et exécutif, de la justice, des autonomies locales, des partis politiques; les institutions informelles sont celles qui fonctionnent selon des normes privées de statut légal ${ }^{47}$.

65 Selon ce courant critique, l'évolution de la Russie pourrait suivre les trois voies discernables suivantes :

66 - l'acceptation graduelle des résultats du fonctionnement du processus électoral et de l'action des institutions officielles ;

67 - la transition au régime sans concurrence politique (voire au régime autoritaire), avec introduction ultérieure des institutions officielles par centralisation du pouvoir et renforcement de l'État ;

68 - l'effondrement du régime, ce qui prendrait la forme d'une escalade des conflits et de la mobilisation active des acteurs de masse ${ }^{48}$.

De plus, ces auteurs mettent en doute les modèles épistémologiques rationalistes dans leur application au post-modernisme ${ }^{49}$. La théorie de la modernisation ainsi que la théorie de la transition qui la continue, supposent un projet téléologique. En outre, deux raisons sont avancées qui rendraient la théorie de la transition inapplicable en Russie. Tout d'abord, le point de départ n'est pas clairement défini. En 1991, le communisme en URSS n'existait plus. Le terme de la transition est encore plus flou, pour autant que le processus n'est pas (encore ?) terminé. Les représentants du courant 
critique proposent donc d'élaborer un nouveau modèle du post-modernisme, apte à éclairer les réalités postcommunistes. Il est évident que dans cette perspective, il conviendrait de modifier le post-modernisme lui-même, sachant que les postmodernistes russes, emboîtant le pas à Baudrillard, le qualifient de «néant ", vu que le post-modernisme nie la connaissance théorique ${ }^{50}$. Mais en admettant même qu'il soit impossible de traiter les réalités de l'époque postcommunistes avec les instruments de la connaissance théorique, on peut toujours tenter, avec profit, de repenser le régime post-soviétique d'une manière post-moderniste ${ }^{51}$.

Pareille interprétation post-moderniste aurait pour but de comprendre dans toute sa complexité la nature des liens noués entre le post-modernisme et la grande diversité des configurations sociales en scène ${ }^{52}$.

En conclusion, nous souhaiterions souligner la variété des approches qui caractérise en Russie le débat actuel en matière de philosophie politique. D'une part, cette variété reflète la difficulté qu'il y a à saisir l'époque post-soviétique dans sa substance et l'opacité attachée aux repères du développement socio-politique. D'autre part, il faut reconnaître que les modèles théoriques actuels restent inadaptés à une appréhension pertinente des procès en cours en Russie sujette, à l'évidence, à des mutations globales.

\section{NOTES}

1. Gadjiev K. S., Polititcheskaia philosophiia [La philosophie politique], M., 1999, 208 p.

2. Pivovarov Iou. S. et Fourkov A. I., « Rousskaiia sistema kak popytka ponimaniia rousskoï istorii » [Le système russe comme essai pour comprendre l'histoire russe], Polis, $\mathrm{n}^{\circ} 4,2001$, p. 37.

3. Gadjiev K. S., op. cit., p. 101.

4. Gadjiev K. S., op. cit., p. 228.

5. Lediaiev V. G., « Vlast : kontseptoual'nyï analiz » [Le pouvoir : analyse conceptuelle], Polis, $\mathrm{n}^{\circ}$ 3, 2000, p. 106.

6. Idem.

7. Lediaiev V. G., « Formy vlasti : tipologitcheskiï analiz » [Les formes du pouvoir : analyse typologique], Polis, $\mathrm{n}^{\circ} 2,2000$, p. 17-18.

8. Ermakov S. V., Kim I. E., Mikhailova T. V., Osetrova E. B., Soukhovolskiï S. V., Vlast'v russkoï iazykovoï I etnitcheskoï kartine mira, [Le pouvoir dans une représentation linguistique et ethnique russe du monde], Moskva, 2004, p. 343.

9. Pchizova S. N., « Democratia i polititcheskiï rynok v sravnitel'noï perspective » [La démocratie et le marché politique dans une perspective comparative], Polis, $\mathrm{n}^{\circ} 2,2000$, p. 31 .

10. Kapoustin B. G., Sovremmenost' kak predmet polititcheskoï teorii, [La contemporanéité comme objet de la théorie politique], M., 1998, p. 283-284.

11. Pchizova S. N., « Democratia i polititcheskiï rynok v sravnitel'noï perspektive », [La démocratie et le marché politique dans une perspective comparative], Polis, $\mathrm{n}^{\circ} 3,2000$, p. 8. 
12. Smorgounov L. V., « Setevoï podkhod k politikie i oupravleniou », [Conception en réseau

de la politique et de la gouver-nance], Polis, $\mathrm{n}^{\circ}$ 3, 2001, p. 111.

13. Zagorodnikov F. N., « Deesposobnost' gosoudarstva i effektivnost' vlasti » [Faculté d'action du gouvernement et efficacité du pouvoir] Nezavisimaia gazeta, 08.04.2000.

14. Kaspe S., Imperia i modernisatsiia. Obscaia model' i rossiïskaia spezifika, [Empire et modernisation. Modèle général et originalité russe], M., 2001, p. 206.

15. Kaspe S., op. cit., p. 30.

16. Volodikhin D., « Medlennyï vzryv imperii » [Lente explosion de l'empire], Imperiia, Sdelaï sam, M., 2001, p. 58 ; Kaspe S. N., « Rossiïskaia federatsiia: stroitel'stvo bez proekta ", [La Fédération russe : une construction sans projet], Polis, $n^{\circ} 5,2000$, p. 62. 17. Volodikhin D., op. cit., p. 58.

18. Kaspe S., Imperiia i modernisatsiia [Empire et modernisation], p. 201-204.

19. Trifonov A. G., Mejouiev B. D., « General-goubernatorstvo v rossï̈skoï sisteme territorial'nogo oupravleniia » [Le gouvernement-général dans le système russe de direction territorial], Polis, $\mathrm{n}^{\circ}$ 5, 2000, p. 19-26.

20. Zoubov A. B., « Ounitarism ili federalism. K voprosou o boudouchtchiem organisatsii gosoudarstvennogo prostranstva Rossii » [Unitarisme ou fédéralisme. À propos de la future organisation de l'espace étatique de la Russie], Polis, $n^{\circ}$ 5, 2000, p. 42.

21. Ibid., p. 52-53.

22. Popov G., « Natsional'nosti v Rossii » [La nationalité en Russie], Nezavisimaia gazeta, 12.04.2000.

23. Osnovy natsional'nosti i federalnykh otnocheniï [Les fondements de la nationalité dans les relations fédérales], 2001, p. 335-336.

24. Ibid., p. 281.

25. « O nazional'nom samotchouvstvii narodov Rossii. O sostoianii i perspektivah gosoudarstvennoï i nazional'noï politiki » [Le sentiment de nationalité des peuples de la Russie. Situation et perspectives de la politique étatique et nationale], Nezavisimaia gazeta, 22.07.2000.

26. Holodnyï V. I. « Rousskoe ponimanie nazii » [La conception russe de la nation], Nezavisimaia gazeta, 22.07.2000.

27. Zakharov A. A., « Ispolnitel'nyï federalism v sovremennoï Rossii » [Le fédéralisme exécutif dans la Russie actuelle], Polis, $\mathrm{n}^{\circ}$ 4, 2001, p. 130.

28. Galkin A. A., Fedosov P. A., Valenteï S. D., Soloveï I. D., « Federalism i poublitchnaia sphera v Rossii » [Le fédéralisme et la sphère publique en Russie], Polis, $\mathrm{n}^{\circ} 4,2001$, p. 133.

29. Gadjiev K. S., Polititcheskaia philosophiia [La philosophie politique], p. 341.

30. Alexeeva T. A., Kapoustine B. G. Pantin I. K., « Perspektivy integrativnoï ideologii » [Perspectives de l'idéologie d'intégration], Polis, n 3, 1997.

31. Malikova O. Iou., « Partiïnye ideologii v Rossii: atribut ili antouraj ? [ [Idéologies de parti : attribut ou entourage ?] Polis, $\mathrm{n}^{\circ}$ 5, 2001, p. 103.

32. Migranian A., Elymanov A., Soloveï B., Opyty gosoudarstvennoï ideologii v sovremennoï Rossii, [Les expériences de l'idéologie d'État dans la Russie actuelle], Rossiia, poisk pouti, M., 1999, p. 63.

33. Volkov Iou. G., « Ideologiia v jisni sovremennogo rossiïskogo obscestva » [L’idéologie dans la vie de la société russe acuelle], Sozial'no-goumanitarnye znaniia, $n^{\circ} 6,1999$. 
34. Soloviev A. I., « Polititcheskaia ideologiia: logika istoritcheskoï evoluzii » [L'idéologie politique : la logique de l'évolution historique], Polis, n 2, 2001, p. 20.

35. Soloviev A. I., op.cit., p. 21.

36. Soloviev A. I., op. cit., p. 22.

37. Ermakov S. V., Kim I. E., Mikhailova T. V., Osetrova E. B., Soukhovolskiï S. V., Vlast'v russkoï iazykovoï I etnitcheskoï kartine mira [Le pouvoir dans une représentation linguistique et ethnique russe du monde], Moskva, 2004, p. 90-91, 117.

38. Preemstvo. Tchto je boudet s Rossieï i s nami [La succession : qu'en sera-t-il de la Russie et de nous ?], M., 2000, p. 23.

39. Dougin A., Rousskaia vesc, [La chose russe], T. 1, M., 2001, p. 72.

40. Erasov B. S., « Soziokul'tournye i geopolititcheskie prinzipy evrasiïstva » [Les principes socioculturels et géopolitiques de l'Eurasie], Polis, n 5, 2001, p. 73.

41. Obsor evrasiïskoï ideologii // Osnovy evrasiïstva [Aperçu de l'idéologie eurasienne//Fondements de l'Eurasie], M. 2002. p. 84-85.

42. Popov G., « Rossiia iscet ideologiiou novogo tysatcheletiïa » [La Russie à la recherche d'une idéologie pour le nouveau millénaire], Nezavisimaia gazeta, 25.08.2000.

43. Goutourov B. A. «Sovremennaia rossiïskaia polititcheskaia ideologiia kak sistema i polititcheskaia real'nost' » [L'idéologie politique russe actuelle comme système et réalité politique], Polis, $\mathrm{n}^{\circ} 3,2001$.

44. Polititcheskiï prozess : osnovnye aspekty $i$ sposoby analisa [Le processus politique : aspects fondamentaux et modes d'analyse], M., 2001, p. 250.

45. Lanzov S. A., « Rossiïskiï istoritcheskiï opyt v svete konsepziï polititcheskoï modernisazii » [L'expérience historique russe à la lumière de la conception de la modernisation politique], Polis, ${ }^{\circ}$ 3, 2001, p. 93-101 ; Volodin A. G. « Grajdanskoie obscestvo i modernisaziia v Rossii » [Les citoyens et la modernisation en Russie], Polis, $\mathrm{n}^{\circ} 3,2000$, p. 114-116.

46. Il'in M. V., Otcherki hronologitcheskoï tipologii [Essai de typologie chronologique], M., 1995 ,

p. 49-51.

47. Gel'man V. Ia., Transformaziia $v$ Rossii : polititcheskiï rejim i demokratitcheskaia opposiziia [Transfor-mation en Russie : régime politique et opposition démocratique], M., 1999, p. 50.

48. Gel'man V. Ia., « Postsovetskie polititcheskie transformazii » [Les transformations politiques postsoviètiques], Polis, $\mathrm{n}^{\circ} 1$, 2001, p. 24-25.

49. Kapoustin B. G., « Konez 'tranzitologii' ? O teoretitcheskom osmyslenii pervogo postkommunistitcheskogo desiatiletiia » [Fin de la «transitologie »? Les interprétations théoriques de la première décennie postcommuniste], Polis, $\mathrm{n}^{\circ} 4,2001$, p. 12-13.

50. Kapoustin B. G., « Postkommunisme kak postsovremennost » [Le postcommisme comme postcontemporanéité], Polis, n 5, 2001, p. 6.

51. Ibid., p. 21.

52. Ibid., p. 12. 


\section{RÉSUMÉS}

Andreï Tyrsenko nous donne un aperçu des débats politiques tels qu'ils se déroulent dans son pays où le pouvoir de l'idéologie est réenvisagé pour permettre l'intégration d'une société en perte de repères. Mais quelle idéologie peut aujourd'hui renforcer le pouvoir de l'État-nation, dans un contexte marqué par son affaiblissement? Les symboles du marché politique, une grande diversité de formes de discours politique, abordée sous l'angle de la communication politique, remplacent les traditions et les idéologies et deviennent les nouveaux repères de l'espace public au potentiel unificateur. Le modèle d'une démocratie dirigée (mélange d'éléments autoritaires) et celui de l'empire restent en Russie les alternatives disponibles pour faire face à la situation de mutation globale actuelle. Le modèle impérial repensé avec les concepts gumiloviens semble avoir de nombreux adeptes. La théorie politique « locale » de Lev Gumilov pallie en effet ce vide d'idéologie politique intégratrice; son succès s'explique en partie par l'attrait de sa construction d'ordre juridique qui hiérarchise les entités politiques entre nations-ethnies et super-ethnies rassemblées dans ce système fédératif et donne une forte dimension terrritoriale au pouvoir, tout en mettant l'accent sur la tolérance interethnique comme principal ciment de cet édifice fédéral.

Andreï Tyrsenko. At which stage is the political debate in Russia?

The author gives an overview of political debates as they take place in his country, where the ideology of power is being considered again to enable integration in a society losing its bearings. A wide diversity of forms of political discourse, approached from the angle of political communication, replaces traditions and ideologies. These forms with their unifying potential are becoming the new landmarks of public space. The model of directed democracy (mix of authoritarian elements) and of empire remain in Russia the available alternatives to face the present situation of global mutation. Re-worked with gumilovian concepts, the imperial model seems to have many adepts. Indeed it makes up for the lack of integrative political ideology. Its success can be partially explained by the attractiveness of its juridical structure which creates a hierarchy of political entities among the ethnic and super ethnic nations gathered together in this federating system and gives a territorial dimension to power whilst stressing inter-ethnic tolerance as the main ciment of this federal structure.

\section{AUTEUR}

\section{ANDREI TYRSENKO}

Maître de conférence, faculté d'histoire de l'université d'État Lomonossov de Moscou, tyrsenko@hotmail.com 